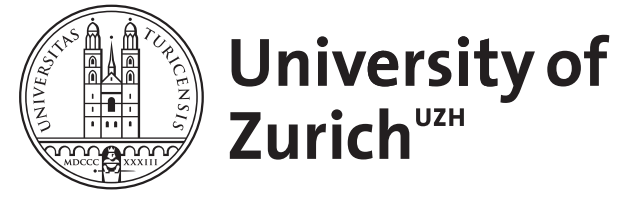
Archive

University of Zurich

University Library

Strickhofstrasse 39

CH-8057 Zurich

www.zora.uzh.ch

Year: 2013

\title{
Leberverfettung: Häufig und nicht ganz harmlos
}

Lang, Christine ; Georgi, Anna ; Gubler, Christoph

DOI: https://doi.org/10.1024/1661-8157/a001420

Posted at the Zurich Open Repository and Archive, University of Zurich

ZORA URL: https://doi.org/10.5167/uzh- 84427

Journal Article

Accepted Version

Originally published at:

Lang, Christine; Georgi, Anna; Gubler, Christoph (2013). Leberverfettung: Häufig und nicht ganz harmlos. Praxis, 102(18):1089-1101.

DOI: https://doi.org/10.1024/1661-8157/a001420 


\section{CME Praxis 08/2013}

\section{Leberverfettung: häufig und harmlos? Oder eben nicht ganz...}

Eine Fettleber liegt per definitionem vor, wenn $>5 \%$ des Lebergewichts aus Fett besteht oder $>50 \%$ der Hepatozyten Fett speichern. Unter dem Begriff nicht-alkoholische Fettlebererkrankung (NAFLD) werden verschiedene Krankheitsentitäten zusammengefasst: die reine nicht-alkoholische Fettleber, die nicht-alkoholische Steatohepatitis (NASH), bei der zusätzlich eine Entzündung des Lebergewebes mit oder ohne fibrotische Veränderungen vorliegt, sowie die Fettleberzirrhose. Als Folge der NAFLD kann es vermutlich auch ohne Zirrhose zur Entwicklung eines hepatozellulären Karzinoms kommen.

Die NAFLD ist mittlerweile die häufigste Lebererkrankung in den Industrienationen. In grösseren Populationsstudien ist die NAFLD für 25-30\% der chronischen Hepatopathien bzw. chronischen Transaminasenerhöhungen verantwortlich. Die Prävalenz der NAFLD nimmt mit dem Alter zu, wobei zunehmend Kinder und Jugendliche davon betroffen sind. Im europäischen Raum haben 20-30\% der Gesamtbevölkerung sonographisch eine Fettleber. Die Prävalenz der NASH wird in Industrieländern analog auf ca. 2-3\% geschätzt. Deutlich höher liegen die Zahlen bei Patienten mit Dyslipidämie, Adipositas und Diabetes mellitus Typ 2, hier weisen $50-70 \%$ eine Fettleber, $20-50 \%$ eine NASH und 2-3\% bereits eine Leberzirrhose auf.

Die NAFLD ist keine isolierte Lebererkrankung, sondern kann als hepatische Manifestation des metabolischen Syndroms verstanden werden. Es gibt eine Vielzahl mit einer NAFLD assoziierter Komorbiditäten (Abb. 1), von denen einige als Prädiktoren für eine NAFLD identifiziert werden konnten, wenngleich noch nicht in allen Fällen ein kausaler Zusammenhang belegt ist. Umgekehrt kann die NAFLD ein unabhängiger Risikofaktor für einige dieser Erkrankungen sein. Von grosser Bedeutung für die Prognose der NAFLD sind nicht nur die Leberspezifischen Komplikationen, sondern auch die „extrahepatischen Manifestationen“ wie kardiovaskuläre Komorbiditäten und Malignome. Bei der NAFLD handelt es sich somit nicht um eine harmlose Fettleber, sondern um eine klinisch ernst zu nehmende und gesundheitsökonomisch äusserst relevante Lebererkrankung.

Pathogenetisch von grosser Bedeutung ist neben der erhöhten Kalorienaufnahme insbesondere die Aufnahme freier Fettsäuren aus dem viszeralen Fett. Die aus dem viszeralen Fett durch Lipolyse freigesetzten Fettsäuren gelangen direkt in die Pfortader und führen so zu einer Verfettung von Leberparenchymzellen. Eine gesteigerte Lipolyse kann unter anderem das Resultat einer fehlenden Suppression durch Insulinresistenz des Fettgewebes sein.

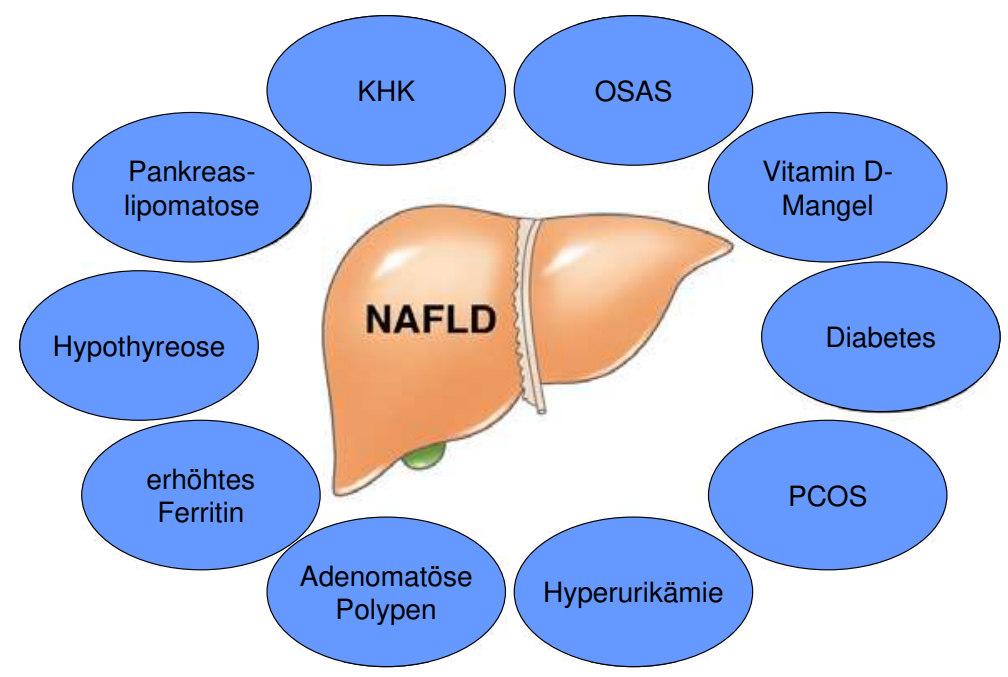

Abb. 1: Mit einer NAFLD assoziierte Komorbiditäten

(modifiziert nach Torres et al., Clin Gastroenterol Hepatol. 2012) 


\section{Im Artikel verwendete Abkürzungen}

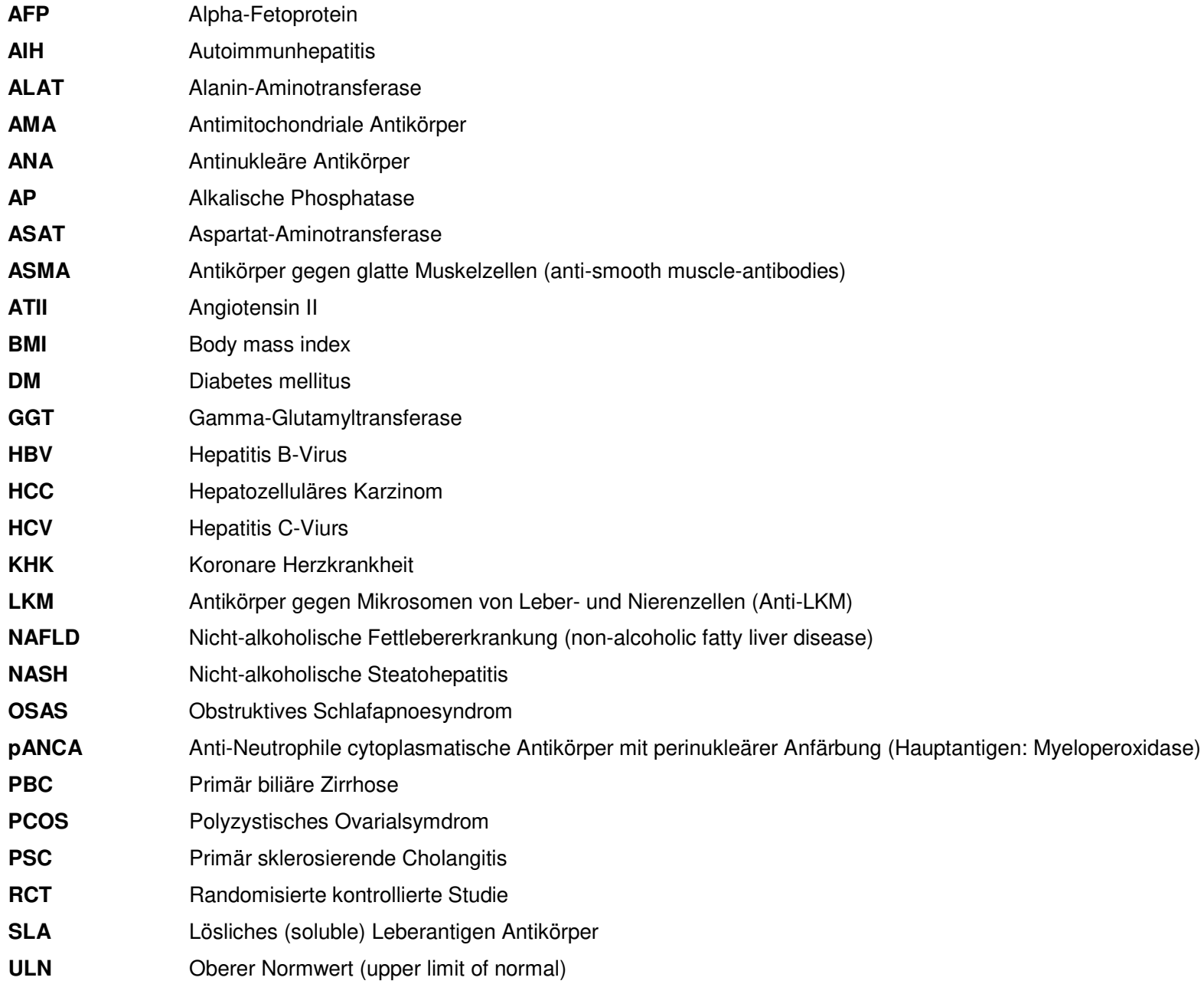




\section{Klinik / Krankheitsverlauf und Prognose}

\section{Klinik}

Die meisten Patienten mit einer NAFLD sind beschwerdefrei oder weisen nur unspezifische Symptome auf, wie rechtsseitige Oberbauchschmerzen, Müdigkeit und Unwohlsein.

In der Regel wird im Rahmen einer routinemässig durchgeführten Laboruntersuchung eine Erhöhung der Leberwerte festgestellt oder in der aus einem anderen Grund erfolgten Bildgebung nebenbefundlich eine hepatische Steatose nachgewiesen.

Die klinischen Befunde sind selten spezifisch. Häufigster Befund ist eine abdominelle Adipositas. Da es sich bei der NAFLD um die hepatische Manifestation des metabolischen Syndroms handelt, wird die Klinik eher durch dessen weitere Organmanifestationen wie Diabetes mellitus oder koronare Herzkrankheit bestimmt. Die typischen Stigmata der chronischen Lebererkrankung (Palmarerythem, Spider naevi, Aszites etc.) sind in der Regel erst bei manifester Leberzirhose zu erwarten.

Laborchemisch können GGT, alkalische Phosphatase und Transaminasen erhöht sein, Bilirubin und Albumin liegen normalerweise im Normbereich. Im Allgemeinen sind die Transaminasen nur leicht bis moderat erhöht (15x ULN). Die ALAT ist meist mehr erhöht als die ASAT mit einem Verhältnis von ASAT : ALAT < 1, im Gegensatz zur alkoholischen Fettlebererkrankung, wo der ASAT : ALAT-Quotient i.d.R. $\geq 2: 1$ beträgt. Obwohl die Höhe der Transaminasen häufig mit dem Ausmass der Entzündung oder Fibrose korreliert, können auch Patienten mit NAFLD oder Zirrhose normale Transaminasen haben.

\section{Krankheitsverlauf und Prognose}

Patienten mit einer NAFLD können im Verlauf eine Fibrose entwickeln, was mit einer schlechteren Prognose einhergeht. Die Progression der Lebererkrankung wird im Wesentlichen vom histologischen Phänotyp bei Diagnosestellung bestimmt. Der stärkste Prädiktor für eine Fibroseprogression ist das Vorliegen einer Entzündungsreaktion. Patienten mit einer reinen Fettleber haben im Gegensatz zu Patienten mit einer NASH nur ein geringes Risiko, eine signifikante Fibrose zu entwickeln. Bei Vorliegen einer NASH ist das Zirrhoserisiko wesentlich höher als bei einer Steatose und liegt zwischen 7 und 25\% innerhalb von 8-10 Jahren. Im Gegensatz dazu entwickelt nur 1-3\% der Patienten mit einer „reinen“ Fettleber innerhalb von 10-20 Jahren eine Leberzirrhose. Wahrscheinlich kommt es bei diesen Patienten zunächst zu einer NASH, eine direkte Progression einer Steatose ohne entzündliche Aktivität zu einer Leberzirrhose scheint eher unwahrscheinlich zu sein. Die Progression zur Fibrose ist sehr variabel und abhängig vom Vorliegen verschiedener weiterer Risikofaktoren (Abb.2).

Hat sich eine Leberzirrhose entwickelt, besteht ein erhöhtes Risiko für das Auftreten Leber-spezifischer Komplikationen. Die Mortalität ist dann erhöht aufgrund von Leberversagen, Sepsis, Varizenblutungen und des Auftretens eines HCC, wobei letzteres selten auch in einer nicht-zirrhotischen Leber beobachtet wurde. 


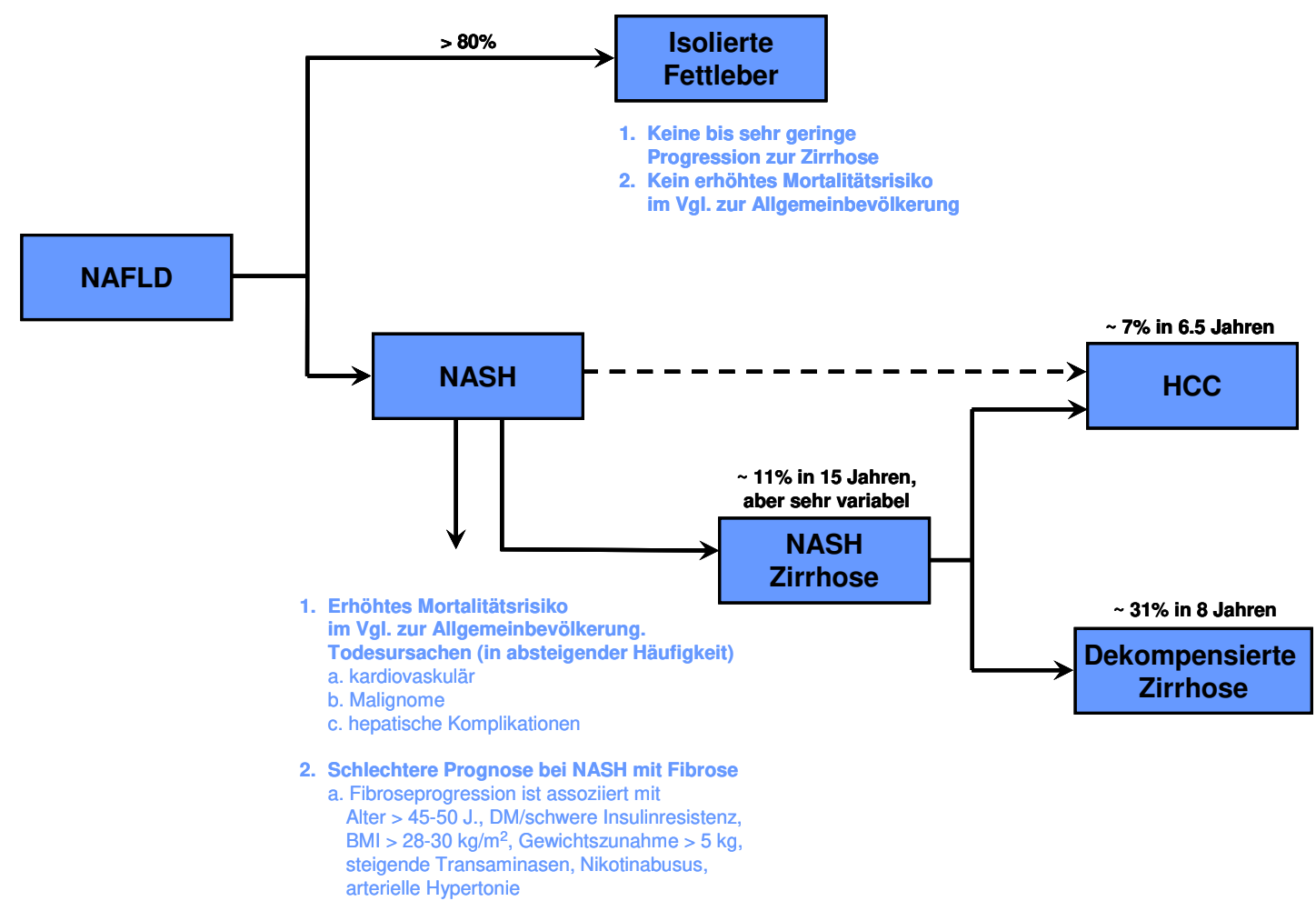

Abb. 2: Natürlicher Verlauf der NAFLD in Abhängigkeit vom histologischen Phänotyp (modifiziert nach Torres et al., Clin Gastroenterol Hepatol. 2012)

Neben den leberspezifischen Komplikationen ist die NAFLD auch ein unabhängiger Prädiktor für das Auftreten anderer Erkrankungen des metabolischen Syndromes. So ist das Risiko, einen Diabetes zu entwickeln, bei Patienten mit einer NAFLD 2.5-fach erhöht.

Die Gesamtmortalität scheint bei Patienten mit einer isolierten Fettleber nicht erhöht zu sein, wohingegen das Vorliegen einer NASH die Mortalität um 35-85\% erhöht. Das erhöhte Mortalitätsrisiko ist in erster Linie auf kardiovaskuläre Erkrankungen zurückzuführen, so ist die kardiovaskuläre Mortalität bei NASH-Patienten auch unabhängig von den klassischen kardiovaskulären Risikofaktoren 2-fach erhöht. Zweithäufigste Mortalitätsursache sind Malignome, gefolgt von Leber-spezifischen Komplikationen an dritter Stelle. 


\section{Diagnose}

Damit die Diagnose einer NAFLD gestellt werden kann, müssen alle folgenden Kriterien erfüllt sein:

(1) Nachweis einer hepatischen Steatose mittels Bildgebung oder Histologie

(2) Ausschluss eines signifikanten Alkoholkonsums

(3) Ausschluss einer anderen Ursache der Steatose

(4) Ausschluss einer koexistenten anderen chronischen Hepatopathie

Entweder wird in der Leberhistologie ein erhöhter Fettanteil oder aber in der Bildgebung (Sonographie oder Computertomographie) eine Steatose diagnostiziert. Letzteres ist oft ein subjektiver Eindruck des Untersuchers. Nun gilt es vor allem mit einer sorgfältigen Anamnese die Ätiologie einzugrenzen (Ausschluss Alkohol, offensichtliche sekundäre Steatose), um die weiterführende Diagnostik gezielt einzusetzen (Tab. 3).

\section{Anamnese}

Zunächst sollte eine komplette Anamnese erhoben werden, in der neben Beschwerden v.a. Risikofaktoren für eine NAFLD (Gewichtsveränderungen, körperliche Inaktivität, Begleiterkrankungen wie Übergewicht/Adipositas, Diabetes mellitus Typ 2, arterielle Hypertonie, Dyslipidämie) erfasst werden sollten. Bei jungen Frauen können Zyklusänderungen und Hirsutismus auf ein polyzystisches Ovarialsyndrom hinweisen.

Daneben muss der Alkoholkonsum, idealerweise auch durch Fremdanamnese, quantifiziert werden. Als signifikanter Alkoholkonsum definiert ist ein täglicher Konsum von $>20 \mathrm{~g}$ bei Frauen und $30 \mathrm{~g}$ bei Männern $(20 \mathrm{~g}$ $=0.5 \mathrm{~L}$ Bier / 0.25 L Wein).

Ferner müssen eine detaillierte Medikamentenanamnese erhoben und Risikofaktoren für andere Lebererkrankungen erfragt werden. Wichtig ist die Frage nach durchgemachter Chemotherapie, da einige dieser Substanzen eine Chemotherapie-assoziierte Steatohepatitis (CASH) induzieren können.

Bei der Erhebung der Familienanamnese sollte der Fokus auf die Häufung metabolischer (Diabetes mellitus) und kardiovaskulärer Erkrankungen sowie der häufigsten genetisch bedingten Hepatopathien, wie hereditäre Hämochromatose, gelegt werden.

\section{Körperliche Untersuchung}

Der Anamnese sollte sich eine komplette körperliche Untersuchung anschliessen, welche das Erfassen von Körpergrösse, Gewicht (BMI) sowie eine Blutdruckmessung beinhaltet. Besonderes Augenmerk sollte auf folgende klinische Zeichen der Leberzirrhose ausgerichtet sein: Spider Naevi, Palmarerythem, Dupytren Kontraktur, kleine noduläre oder vergrösserte Leber, palpable Splenomegalie. Bei fortgeschrittener Lebererkrankung kommen Ödeme, Aszites und Ikterus dazu.

\section{Labor}

Die Labordiagnostik dient in erster Linie dem Ausschluss von Differentialdiagnosen.

Gesucht werden müssen Virushepatitiden (Hepatitis B und C), autoimmune Leber- und Gallenwegserkrankungen (AlH, PSC, PBC) und chronische Speichererkrankungen der Leber bei spezifischen klinischen Hinweisen (Hämochromatose, M. Wilson, Alpha-1-Antitrypsinmangel) (Tab. 1).

Standardmässig bestimmt man ein grosses Blutbild, die Transaminasen, Cholestaseparameter und Lebersyntheseparameter (Albumin, Bilirubin, Quick).

Entsprechend der häufig mit einer NAFLD assoziierten Komorbiditäten (metabolisches Syndrom, Hyperurikämie) empfiehlt sich die Bestimmung von Lipidprofil, HbA1c, TSH und Harnsäure. Ein erhöhtes Ferritin mit normaler Transferrin-Sättigung passt sehr gut zu einer NASH und soll nicht zu einer genetischen Abklärung betreffend Hämochromatose führen (Tab. 2). 
Tab. 1: Sinnvolle Labordiagnostik

\begin{tabular}{|l|}
\hline - Transaminasen \\
- GGT, AP \\
- Bilirubin \\
- Albumin \\
\hline - Blutbild \\
\hline - Quick \\
\hline - Triglyceride \\
- Cholesterin (gesamt, LDL) \\
- HDL-Cholesterin \\
\hline - Nüchternglucose \\
- HbA1c \\
\hline - TSH \\
\hline - Harnsäure \\
\hline - Hepatitis C (Anti-HCV-AK) \\
- Hepatitis B (Anti-HBc-AK, HBs-Ag) \\
- Hepatitis A (Hepatitis A IgG) \\
\hline - Ferritin \\
- Transferrin-Sättigung \\
\hline - Autoantikörper (ANA, LKM, ASMA, SLA, AMA/AMA-M2, pANCA) \\
- Immunglobuline (IgG, IgM, IgA) \\
\hline - Serum-Caeruloplasmin \\
- Kupfer im 24h-Sammelurin \\
\hline - Alpha-1-Antitrypsin \\
\hline
\end{tabular}

Tab. 2: Typisch pathologische Laborparameter

\begin{tabular}{|l|}
\hline - Transaminasen \\
- GGT \\
\hline - Triglyceride \\
- Cholesterin (gesamt, LDL) \\
- HDL-Cholesterin \\
\hline - Nüchternglucose \\
- HbA1c \\
\hline - Ferritin \\
\hline - TSH \\
\hline - Harnsäure \\
\hline
\end{tabular}

\section{Bildgebung}

Geeignete Untersuchungsmodalitäten sind Sonographie, Computertomographie und MRI, mit Hilfe derer der Nachweis einer hepatischen Steatose ab einem Fettgehalt von > 20-30\% gelingt. Eine Aussage über das Vorliegen einer Steatohepatitis oder den Fibrosegrad ist mittels Bildgebung nicht möglich.

Verfahren der ersten Wahl ist die Sonographie, deren Sensitivität jedoch bei adipösen Patienten und Meteorismus eingeschränkt sein kann. Aufgrund der Abhängigkeit vom Untersucher und der eingeschränkten Reproduzierbarkeit, ist sonographisch eine zuverlässige Quantifizierung der Steatose nicht möglich.

Die breite Anwendung von CT bzw. MRI, insbesondere für die Verlaufsbeurteilung, ist limitiert durch die Strahlenbelastung bzw. hohen Kosten.

\section{Leberbiopsie}

Goldstandard für die Diagnose ist die Leberbiopsie. Sie erlaubt neben der Diagnose einer NAFLD die Differenzierung zwischen Fettleber und NASH, die Feststellung des Fibrosestadiums und kann Auskunft über eine vorliegende hepatologische Komorbidität geben. Dadurch ist eine Aussage möglich über Prognose und Dringlichkeit einer Therapie. Im klinischen Alltag ist eine Leberbiopsie jedoch nur sinnvoll, wenn sich aus dem histologischen Befund auch therapeutische Konsequenzen ergeben, welche bei einer NASH noch limitiert sind. Nach Ausschluss anderer häufiger Hepatopathien führt eine Leberbiopsie nur in $20-30 \%$ der Fälle zu einer Änderung der ursprünglichen Arbeitsdiagnose.

\section{Nicht-invasive Alternativen zur Leberbiopsie}

Um die Abklärung einer grossen Patientenzahl zu vereinfachen, sind zur Abschätzung des prognostisch relevanten Fibrosegrades nicht-invasive Methoden erforderlich. Die Kombination aus Serummarkern und bildgebenden Verfahren wird empfohlen, um die Leberbiopsie auf Patienten zu begrenzen, bei denen die 
Resultate nicht eindeutig oder widersprüchlich sind oder bei denen der Verdacht auf eine fortgeschrittene Fibrose besteht.

Verschiedene Scoring-Systeme, basierend auf klinischen und laborchemischen Parametern wurden entwickelt, um NAFLD-Patienten mit einer fortgeschrittenen Fibrose zu identifizieren. Am besten untersucht ist der NAFLDFibrose-Score, bei welchem multiple Variablen (Alter, ASAT, ALAT, Thrombozytenzahl, BMI,

Nüchternglucose/Diabetes, Albumin und das ASAT/ALAT-Verhältnis) in einem komplexen Algorithmus für die Berechnung genutzt werden. Ein NAFLD-Fibrose-Score Rechner ist im Internet verfügbar (http://gihep.com/calculators/hepatology/nafld-fibrosis-score/). Ein Score <-1.455 schliesst eine signifikante Fibrose praktisch aus, ein Score $>0.676$ spricht für eine signifikante Fibrose.

Die transiente Elastographie (FIBROSCAN ${ }^{\circledR}$ ) ermöglicht eine Messung der Lebersteifigkeit, welche mit dem Fibrosegrad korreliert. Bei einem Wert $<7.0 \mathrm{kPa}$ liegt keine oder allenfalls eine milde Fibrose vor, ein Wert von 7.0-9.5 kPa spricht für eine signifikante und ein Wert von 9.5-12.5 kPa für eine fortgeschrittene Fibrose, bei einem Wert $>12.5 \mathrm{kPa}$ ist eine Zirrhose naheliegend. Allerdings führen auch Steatose, Cholestase,

Rechtsherzinsuffizienz und eine akute Hepatitis zu einer erhöhten Lebersteifigkeit und bei adipösen Patienten ist die Anwendung aufgrund technischer Probleme limitiert.

\section{Differentialdiagnose}

Differentialdiagnostisch müssen von der primären NAFLD, die sekundären Formen abgegrenzt werden. Tabelle 3 gibt eine Übersicht über Ursachen einer sekundären Leberzellverfettung.

Tab. 3: Ursachen einer sekundären hepatischen Steatose (modifiziert nach Loria et al., Dig Liver Dis. 2010)

\begin{tabular}{|c|c|c|c|}
\hline Nutritiv & Medikamente/Drogen & Metabolisch/genetisch & Andere \\
\hline $\begin{array}{l}\text { - Malnutrition } \\
\text { - Total-parenterale } \\
\text { Ernährung } \\
\text { - Schneller } \\
\text { Gewichtsverlust } \\
\text { - Chirurgische Eingriffe } \\
\text { am Magen-Darmtrakt } \\
\text { incl. bariatrischer } \\
\text { Chrirurgie } \\
\text { - Short Bowel Syndrom }\end{array}$ & $\begin{array}{l}\text { - Glukokortikoide } \\
\text { - Östrogenpräparate } \\
\text { - Ca-Antagonisten } \\
\text { (Nifedipin/Diltiazem) } \\
\text { - Amiodaron } \\
\text { - Tamoxifen } \\
\text { - Tetrazykline } \\
\text { - Trimethoprim/Sulfamethoxazol } \\
\text { - Methrotrexat } \\
\text { - Valproinsäure } \\
\text { - HAART } \\
\text { - Kokain } \\
\text { - Ekzessiver Alkoholkonsum } \\
\text { - CASH }\end{array}$ & $\begin{array}{l}\text { - Lipodystrophie } \\
\text { - Dysbetalipoproteinämie } \\
\text { - Weber-Christian- } \\
\text { Erkrankung } \\
\text { - Wolmans-Erkrankung } \\
\text { - Lipidspeicherkrankheiten } \\
\text { - Akute Fettleber der } \\
\text { Schwangerschaft } \\
\text { - Morbus Wilson } \\
\text { - Hereditäre } \\
\text { Hämochromatose (HFE) }\end{array}$ & $\begin{array}{l}\text { - Chron. entzündliche } \\
\text { Darmerkrankungen } \\
\text { - Dünndarmdivertikulose mit } \\
\text { bakterieller } \\
\text { Überwucherung } \\
\text { - HIV } \\
\text { - Virale Hepatitis } \\
\text { - Phosphor } \\
\text { - Erdölchemikalien } \\
\text { - Pilzvergiftungen } \\
\text { - Organische Lösungsmittel } \\
\text { - Bacillus cereus }\end{array}$ \\
\hline
\end{tabular}


Ziel ist es, Patienten mit einer fortgeschrittenen Lebererkrankung, definiert als NASH mit Fibrose oder Zirrhose, zu identifizieren, da sich bei diesen Patienten neben der prognostischen Information auch Konsequenzen bzgl. Management und Therapie ergeben.

Ein generelles Screening von Patienten, auch bei positiver Familienanamnese für eine NAFLD, kann aufgrund der aktuellen Datenlage nicht empfohlen werden.

Bei Risikopopulationen (z.B. Adipositas, Diabetes mellitus, Dyslipidämie, PCOS etc.) ist die Prävalenz einer NASH deutlich höher als in der Allgemeinbevölkerung, insbesondere wenn die ALAT erhöht ist. Bei diesen Patienten kann eine Abklärung mittels Bestimmung der Leberwerte und Sonographie erwogen werden, da sich aus der Diagnose eventuell Konsequenzen hinsichtlich des Managements ergeben können. Eine Leberbiopsie ist in diesen Fällen nur bei entsprechender Risikokonstellation nach nicht-invasiver Abklärung erforderlich (s.a. Abklärungsstrategie), sofern sich daraus therapeutische Konsequenzen ergeben.

\section{Abklärungsstrategie}

Besteht der Verdacht auf eine NAFLD, sollten zunächst Differentialdiagnosen anamnestisch und laborchemisch ausgeschlossen werden und eine nicht-invasive Risikostratifizierung für das Vorliegen einer Fibrose vorgenommen werden.

Bislang gibt es keinen klaren Konsensus, wann eine Leberbiopsie erfolgen sollte, so dass die Entscheidung für eine invasive Abklärung individuell getroffen werden muss.

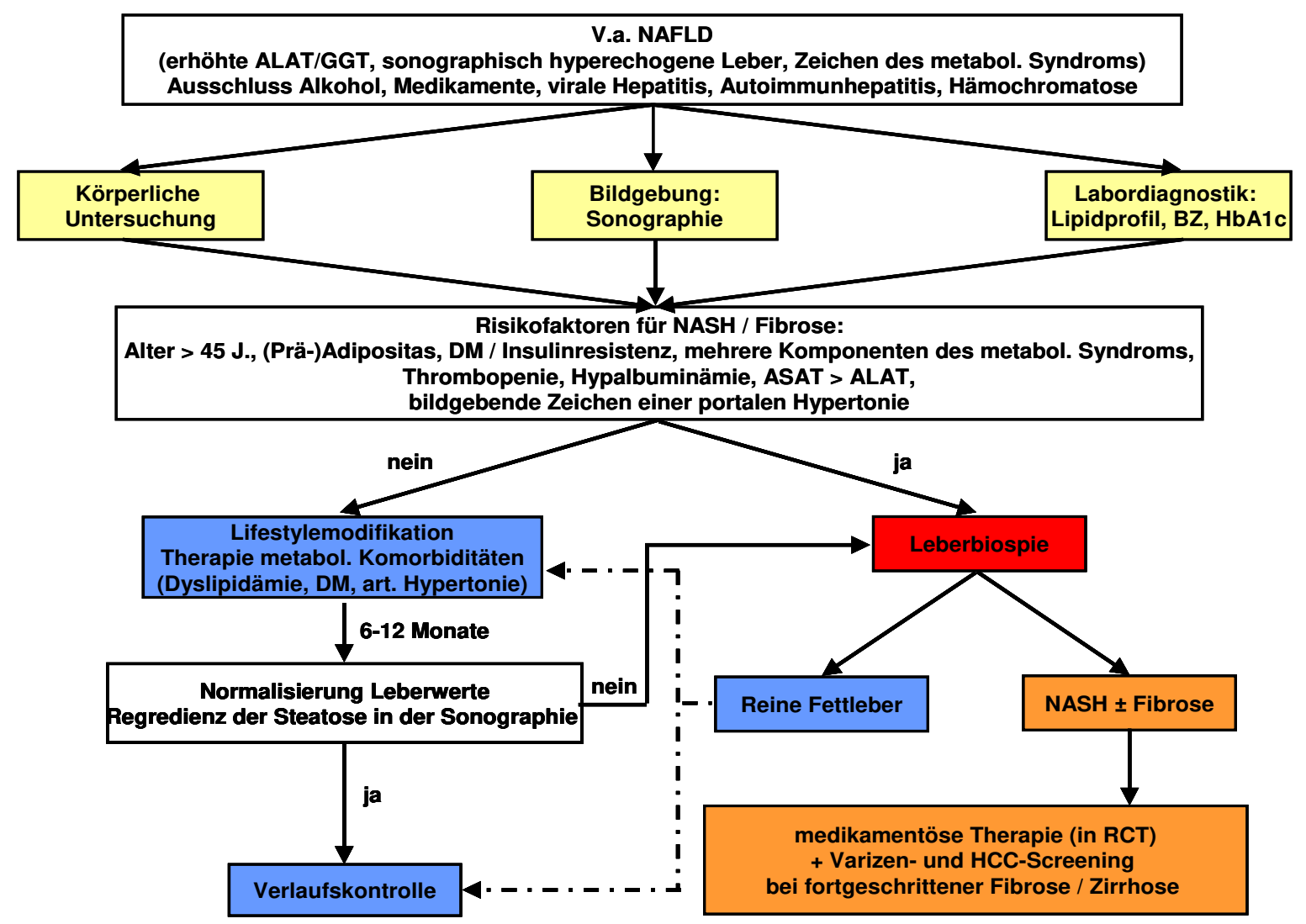

Abb. 3: Abklärungsstrategie bei NAFLD (modifiziert nach Loria et al., Dig Liver Dis. 2010) 
Nicht durchgeführt werden sollte eine Biopsie bei Patienten mit vorangegangener Änderung des Körpergewichts. Bei Patienten, bei denen keine Zeichen einer fortgeschrittenen Fibrose vorhanden sind, ist es gerechtfertigt, auf eine Biopsie zu verzichten und die Patienten zu einer Lifestyle-Modifikation zu motivieren. Bei persistierender Hepatopathie nach 6 Monaten sollte nach Abwägen des Risikos für eine fortgeschrittene Fibrose (klinische, laborchemische Parameter, NAFLD-Fibrose-Score, FIBROSCAN ${ }^{\circledR}$ ) und unter Berücksichtigung von Komorbiditäten und individueller Motivation des Patienten eine Biopsie erwogen werden.

Tab. 4: Mögliche Indikationen für eine Leberbiopsie

\begin{tabular}{|l|l|}
\hline Indikatoren einer Biopsie & Kommentar \\
\hline - Resultate der nicht-invasiven Diagnostik nicht & - Diagnosessicherung \\
eindeutig & - Ausschluss anderer Hepatopathien \\
\hline $\begin{array}{l}\text { - kutane Stigmata einer chronischen Hepatopathie } \\
\text { - Splenomegalie }\end{array}$ & - Bestätigung einer vermuteten Zirrhose \\
- Zytopenie & \\
- Hypalbuminämie, Hyperbilirubinämie & \\
\hline - Hyperferritinämie bei patholog. Transferrin-Sättigung & - Ausschluss Hämochromatose \\
\hline $\begin{array}{l}\text { - Alter > 45J. + Diabetes mellitus Typ 2 oder Adipositas } \\
\text { - multiple Komponenten des metabolischen Syndroms } \\
\text { - pathologischer NAFLD-Fibrose-Score } \\
\text { - erhöhte Leberstiffness in der transienten Elastographie }\end{array}$ & - Risikofaktoren für fortgeschrittene Fibrose \\
\hline $\begin{array}{l}\text { - persistierende Transaminasenerhöhung trotz } \\
\text { erfolgreicher Lifestylemodifikation }\end{array}$ & - Ausschluss Differentialdiagnose \\
\hline
\end{tabular}




\section{而}

\section{Therapie}

Zur Prävention einer zusätzlichen Hepatopathie sollten alle Patienten mit einer NAFLD, bei denen serologisch keine Immunität besteht, aktiv gegen Hepatitis A und B geimpft werden.

Therapeutisch müssen neben einer Behandlung der Lebererkrankung v.a. auch assoziierte metabolische Komorbiditäten identifiziert und adäquat behandelt werden.

Für die NAFLD selbst existiert bislang keine kausale Evidenz-basierte medikamentöse Therapie, im Vordergrund stehen nicht-pharmakologische Massnahmen und die Behandlung der Risikofaktoren.

\section{Nicht-pharmakologische Therapienansätze}

Therapeutisch an erster Stelle steht eine Gewichtsreduktion durch Ernährungsumstellung und vermehrte körperliche Aktivität (Lifestylemodifikation). Bereits ein moderater Gewichtsverlust mit nur geringem Effekt auf das Körperfett resultiert in einer signifikanten Reduktion des hepatischen Fettgehaltes. Angestrebt werden sollte eine Gewichtsabnahme von 0.5-1 kg/Woche, ein schnellerer Gewichtsverlust ist mit einer Verschlechterung der Hepatopathie assoziiert. Auf der Basis verschiedener Studien wird von internationalen Fachgesellschaften eine Gewichtsreduktion um 7\% des Ausgangsgewichts empfohlen, wobei keiner speziellen Diätform der Vorzug zu geben ist. Allerdings sollte der Konsum von Fruktose auf ein Minimum beschränkt werden, da ein hoher Fruktosekonsum zur Insulinresistenz und NAFLD beiträgt.

Für einen vollständigen Verzicht auf Alkohol gibt es keine ausreichende Evidenz, allerdings sollte der Alkoholkonsum auf $<20 \mathrm{~g} / \mathrm{d}$ beschränkt werden.

Körperliche Aktivität führt auch ohne relevante Gewichtsabnahme zu einer Reduktion der hepatischen Steatose und sollte, wann immer möglich, in den Alltag integriert werden. Empfohlen wird ferner wöchentlich eine körperliche Aktivität über $2.5 \mathrm{~h}$ in moderater Intensität (z.B. schnelles Gehen) und über $75 \mathrm{~min}$ in hoher Intensität (z.B. Joggen).

\section{Medikamentöse Therapienansätze}

Bei der Subgruppe von Patienten mit einer NASH wurden verschiedene pharmakologische Therapieansätze evaluiert.

Ein möglicher Therapieansatz sind Insulinsensitizer. Während Metformin keinen signifikanten Effekt auf die Leberhistologie zeigte, führte Pioglitazon während der Behandlungsdauer histologisch zu einer Verbesserung von Steatose und Entzündung. Allerdings kann der routinemässige Einsatz dieser Medikamente zur Behandlung einer NASH aufgrund der signifikanten Nebenwirkungen nicht empfohlen werden.

Vitamin E führte in einer Dosierung von $800 \mathrm{IE} / \mathrm{d}$ histologisch zu einer Regredienz von Steatose und Entzündung. Allerdings gibt es aus Beobachtungsstudien Hinweise, dass hohe Vitamin E-Dosen ( $>400 \mathrm{IE} / \mathrm{d}$ ) mit einer erhöhten Gesamtmortalität einhergehen. Bei Patienten mit histologisch gesicherter NASH erscheint ein Therapieversuch mit 400-800 E/d diskutabel, sofern kein Diabetes mellitus und keine Zirrhose vorhanden sind.

Eine Supplementation mit Omega-3-Fettsäuren führte in einigen Studien ebenfalls zu einer Reduktion des hepatischen Fettgehaltes. Eine allgemeine Empfehlung für Omega-3-Fettsäuren zur Behandlung einer NAFLD gibt es nicht. Da in erster Linie kardiovaskuläre Erkrankungen für die erhöhte Mortalität bei NAFLD-Patienten verantwortlich sind, kann eine Supplementation jedoch nicht zuletzt aufgrund des kardiovaskulären Benefits und als First-Line Therapie einer Hypertriglyceridämie erwogen werden.

Zum Einsatz von Statinen und Ezetimib zur Therapie der NASH gibt es keine Evidenz. Zur Behandlung einer Dyslipidämie können diese Medikamente, unter regelmässiger Transaminasenkontrolle, jedoch ohne erhöhtes Risiko eines medikamentös-toxischen Leberschadens auch bei einer NAFLD eingesetzt werden. 
Die ebenfalls in Studien untersuchten Medikamente wie Orlistat, Ursodesoxycholsäure, Probucol, Betaine Pentoxifyllin und Angiotensin II (ATII)-Antagonisten können aufgrund der aktuellen Datenlage nicht zur Therapie einer NASH empfohlen werden. ATII-Antagonisten können zur Behandlung einer arteriellen Hypertonie bei $\mathrm{NASH}-$ Patienten in Betracht gezogen werden.

Ein Vitamin D-Mangel sollte aufgrund der positiven Assoziation mit einer NAFLD sowie den auch extrahepatisch nachteiligen Effekten entsprechend substituiert werden. Es gibt Hinweise für eine positiven therapeutischen Einfluss von Vitamin D auf die NASH.

Zusammengefasst kann zur Zeit noch keine medikamentöse Therapie der NAFLD ausserhalb von Studien empfohlen werden.

\section{Follow up}

Aufgrund des erhöhten Risikos für einen Diabetes mellitus Typ 2 und das Auftreten eines metabolischen Syndroms bzw. seiner Komponenten sollte bei Patienten mit einer NAFLD in regelmässigen Abständen von 6-12 Monaten eine nicht-invasive Evaluation des kardiovaskulären Risikos (Laboruntersuchung, Klinik) erfolgen.

Bei Patienten mit einer NAFLD, insbesondere bei histologisch gesicherter NASH, sind jährliche Verlaufskontrollen mit Bestimmung von Transaminasen und Lebersyntheseparametern indiziert und sollten bei nachgewiesener Fibrose eine Elastographie beinhalten. Handelt es sich histologisch um eine „reine“ Fettleber, kann aufgrund des benignen Verlaufs auf eine engmaschige Surveillance verzichtet werden.

Hat sich bereits eine Leberzirrhose entwickelt, ist ein Screening auf Ösophagusvarizen und eine HCC-

Surveillance mittels Sonographie und AFP-Bestimmung in 6-monatlichen Intervallen indiziert.

Die NAFLD geht mit einem erhöhten Risiko auch extra-hepatischer Malignome einher. Ein generelles Screening wird jedoch nicht empfohlen, das Screening sollte vielmehr dem individuellen Malignomrisiko unter Berücksichtigung auch der Familienanamnese angepasst werden. 


\section{Fallbericht}

U

C. Anamnese des Patienten

JL: Zuweisung des Patienten vom Hautarzt zur Abklärung erhöhter Leberwerte, welche im Rahmen einer Laborkontrolle vor Therapiebeginn mit Alitretinoin (VitaminA-Säure-Derivat) aufgrund eines chronischen Handekzems aufgefallen waren. Der Patient berichtete von einer chronischen Müdigkeit seit 4-5 Monaten ohne weitere Beschwerden. Insbesondere abdominelle Beschwerden, Übelkeit, Stuhlunregelmässigkeiten, Meläna, Hämatochezie, Ikterus und Pruritus wurden verneint.

SysA: Kein Fieber, Appetit nicht reduziert, keine Gewichtsveränderungen, kein regelmässiger Sport, körperliche Aktivität im Alltag gering. Noxen: kein Nikotin, Alkohol: sehr selten (max. 1 Bier/Monat).

Medikamente: Nutraplus Crème, Elocom Crème, keine Medikamente per os

FA: bzgl. Lebererkrankungen bland, Mutter: arterielle Hypertonie; Vater: Dyslipidämie, DM Typ 2, Myokardinfarkt mit $59 \mathrm{~J}$

PA: chronisches hyperkeratotisches irritativ-toxisches Handekzem, ansonsten bland

SozA: verheiratet, 2 Söhne (7J., 5J.), Elektriker

\section{Weg zur Diagnose/Kommentar}

Wie die meisten Patienten mit einer nichtalkoholischen Fettlebererkrankung war auch unser Patient zum Zeitpunkt der Diagnosestellung asymptomatisch. Es bestand lediglich eine chronische Müdigkeit als unspezifisches Symptom.

Auffallend war eine körperliche Inaktivität. Ein signifikanter Alkoholkonsum $>30 \mathrm{~g} / \mathrm{d}$ wurde verneint

Eine systemische Steroidwirkung der Elocom Crème kam als mögliche medikamentös-toxische Ursache in Betracht.

Weg zur Diagnose/Kommentar

Auffallend bei der körperlichen Untersuchung waren eine Präadipositas und eine leichte Hypertonie 1. Grades. 


\begin{tabular}{|c|c|c|c|c|}
\hline \multicolumn{4}{|l|}{ Routinelabor } & \multirow{12}{*}{$\begin{array}{l}\text { Im Routinelabor fand sich eine deutliche Erhöhung } \\
\text { der Transaminasen (ALAT bis } 4 \times \text { XLN, ALAT >> } \\
\text { ASAT), weniger auch der GGT. } \\
\text { Die Lebersyntheseparameter waren normal. }\end{array}$} \\
\hline Hämoglobin & \multicolumn{2}{|l|}{$16.1 \mathrm{~g} / \mathrm{dl}$} & $(13.4-17.0)$ & \\
\hline Thrombozyten & \multicolumn{2}{|l|}{$311 \times 10^{9} / 1$} & $(143-400)$ & \\
\hline Leukozyten & \multicolumn{2}{|l|}{$5.24 \times 10^{9} / 1$} & $(3.0-9.6)$ & \\
\hline Quick & \multicolumn{2}{|l|}{$99 \%$} & $(70-120)$ & \\
\hline INR & \multicolumn{2}{|l|}{1.0} & $(<1.2)$ & \\
\hline ASAT (GOT) & $92 \mathrm{U} / \mathrm{I}$ & $\uparrow \uparrow$ & $(<50)$ & \\
\hline ALAT (GPT) & $206 \mathrm{U} / \mathrm{I}$ & $\uparrow \uparrow \uparrow$ & $(10-50)$ & \\
\hline GGT & $77 \mathrm{U} / \mathrm{l}$ & $\uparrow$ & $(8-61)$ & \\
\hline Alk. Phosphatase & \multicolumn{2}{|l|}{$104 \mathrm{U} / \mathrm{I}$} & $(40-129)$ & \\
\hline Albumin & \multicolumn{2}{|l|}{$45 \mathrm{~g} / \mathrm{l}$} & $(40-49)$ & \\
\hline Bilirubin, total & \multicolumn{2}{|l|}{$5 \mu \mathrm{mol} / \mathrm{l}$} & $(<21)$ & \\
\hline Nüchtern-Glucose & \multicolumn{2}{|l|}{$4.7 \mathrm{mmol} / \mathrm{l}$} & $(<5.6)$ & Auffallend im Routinelabor waren ferner eine \\
\hline HbA1c & \multicolumn{2}{|l|}{$6.2 \%$} & $(<5.9)$ & $\begin{array}{l}\text { prädiabetische Stoffwechsellage und eine } \\
\text { Dyslipidämie mit tiefem HDL-Cholesterin und }\end{array}$ \\
\hline Cholesterin & \multicolumn{2}{|l|}{$4.6 \mathrm{mmol} / \mathrm{l}$} & $(<5.0)$ & Hypertriglyceridämie. \\
\hline HDL-Cholesterin & \multicolumn{2}{|c|}{$0.85 \mathrm{mmol} / \mathrm{l} \downarrow$} & $(>1.0)$ & \\
\hline LDL-Cholesterin & \multicolumn{2}{|c|}{$2.6 \mathrm{mmol} / \mathrm{l}$} & $(<3.0)$ & \\
\hline Triglyceride & \multicolumn{2}{|c|}{$2.60 \mathrm{mmol} / \mathrm{l} \uparrow$} & $(<1.7)$ & \\
\hline Harnsäure & \multicolumn{2}{|l|}{$360 \mu \mathrm{mol} / \mathrm{l}$} & $(120-420)$ & Eine Hyperurikämie und Hypothyreose konnten \\
\hline TSH & \multicolumn{2}{|l|}{$2.77 \mathrm{mU} / \mathrm{l}$} & $(0.30-3.18)$ & ausgeschlossen werden. \\
\hline \multicolumn{5}{|l|}{ Erweitertes Labor } \\
\hline Coerulosplasmin & \multicolumn{2}{|l|}{$242 \mathrm{mg} / \mathrm{l}$} & $(200-600)$ & $\begin{array}{l}\text { Das normale Coeruloplasmin sprach ebenso wie } \\
\text { die Klinik gegen einen M. Wilson. }\end{array}$ \\
\hline Eisen & \multicolumn{2}{|l|}{$17.4 \mu \mathrm{mol} / \mathrm{l}$} & $(11.0-28.0)$ & Die Ferritinerhöhung war hinweisend auf eine \\
\hline Ferritin & $1027 \mu \mathrm{g} / \mathrm{l}$ & $\uparrow \uparrow \uparrow$ & $(30-400)$ & Entzündung und Destruktion von Hepatozyten. \\
\hline Transferrin & $31 \mu \mathrm{mol} / \mathrm{l}$ & & $(25-50)$ & Eine Hämochromatose war bei normaler \\
\hline Transferrin-Sättigung & $29 \%$ & & $(20-55)$ & Transferrinsättigung unwahrscheinlich. \\
\hline$\alpha-1$ Antitrypsin & $1.42 \mathrm{~g} / \mathrm{l}$ & & $(0.90-2.00)$ & $\begin{array}{l}\text { Ein } \alpha-1 \text { Antitrypsin-Mangel konnte ausgeschlossen } \\
\text { werden. }\end{array}$ \\
\hline Immunglobulin IgA & $4.2 \mathrm{~g} / \mathrm{l}$ & $\uparrow$ & $(0.7-4.0)$ & Das leicht erhöhte IgA war als Hinweis auf eine \\
\hline Immunglobulin IgM & $1.1 \mathrm{~g} / \mathrm{l}$ & & $(0.4-2.3)$ & mögliche toxische Leberschädigung zu werten. \\
\hline Immunglobulin IgG & $14.8 \mathrm{~g} / \mathrm{l}$ & & $(7.0-16.0)$ & Die serologischen Abklärungen hinsichtlich \\
\hline ANA & negativ & & $(<1: 10)$ & Autoimmunhepatitis, primär sklerosierender \\
\hline Anti-glatte Musk. (ASMA) & negativ & & $(<1: 10)$ & Cholangitis und primär biliärer Zirrhose waren \\
\hline Anti-LKM & 2 Units & & $(<20)$ & negativ. \\
\hline Anti-SLA & $0 \mathrm{E} / \mathrm{ml}$ & & $(<20)$ & \\
\hline Anti-Mitochondrien (AMA) & negativ & & $(<1: 10)$ & \\
\hline ANCA & negativ & & $(<20)$ & \\
\hline p-ANCA & $0 \mathrm{E} / \mathrm{ml}$ & & $(<5)$ & \\
\hline
\end{tabular}




\begin{tabular}{|c|c|}
\hline 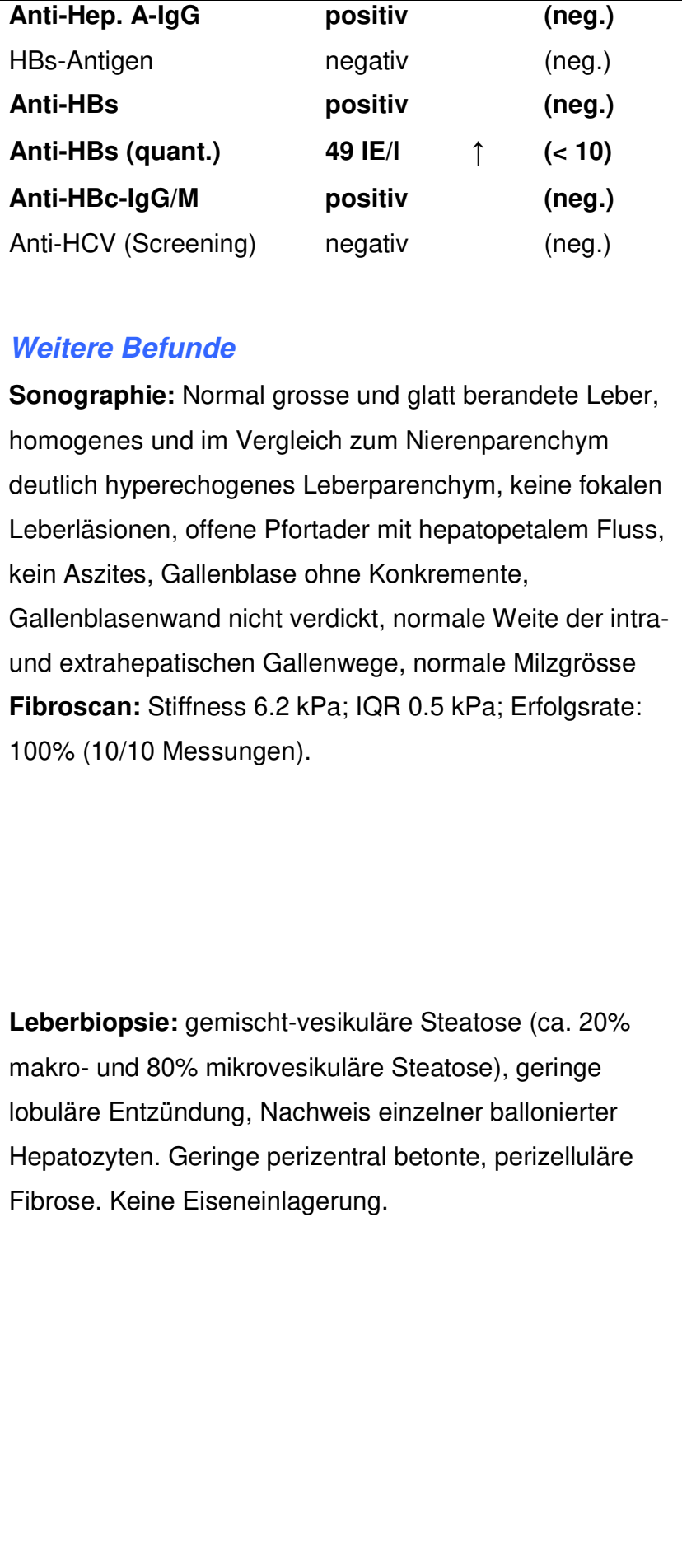 & $\begin{array}{l}\text { Die Lebersteifigkeit von } 6.2 \text { kPa sprach gegen eine } \\
\text { signifikante Fibrose, eine milde Fibrose war bei } \\
\text { diesem Wert aber möglich. Die Messgenauigkeit } \\
\text { war mit einer nur geringen Schwankungsbreite der } \\
\text { Einzelwerte (tiefer IQR) gut. } \\
\text { Histologisch fand sich eine vorwiegend } \\
\text { mikrovaskuläre Steatose, was zu einer NAFLD } \\
\text { passt. Die gering auch makrovesikuläre Steatose } \\
\text { sprach für eine zusätzlich leichte toxische } \\
\text { Komponente. } \\
\text { Das Grading der NAFLD mittels NAFLD-Activity- } \\
\text { Score (NAS), bei dem die } 3 \text { histologischen } \\
\text { Merkmale Steatose (0-3), lobuläre Entzündung (0- } \\
\text { 2) und hepatozelluläre Ballonierung (0-2) evaluiert } \\
\text { werden, ergab einen Score von } 3 \text { (NAS 1-2: keine } \\
\text { NASH/NAFLD; NAS 3-4: Borderline-NASH; } \\
\text { NAS 5-8: Vorliegen einer NASH). Die geringe } \\
\text { Fibrose passte zum Muster einer NASH. }\end{array}$ \\
\hline
\end{tabular}

II

Therapie des Patienten

Die Therapie bestand aus einer Änderung des Lebenstils mit Gewichtsreduktion durch gesteigerte körperliche Aktivität und Diät. Der Patient wurde ausführlich über diese nicht-medikamentöse Basistherapie informiert. Es erfolgte eine Ernährungsberatung mit Analyse der bisherigen Essgewohnheiten und ein Diätplan bestehend aus einer energiereduzierten Mischkost mit verringertem Kohlenhydrat- und Fettanteil sowie einem niedrigen Gehalt an gesättigten Fettsäuren und Fruktose wurde erstellt. Mit physiotherapeutischer Hilfe wurde ein Trainingskonzept mit regelmässigem Ausdauertraining und Integration von körperlicher Aktivität in den Alltag erarbeitet. Mit Hilfe dieser Massnahmen war es dem Patienten gelungen, sein Gewicht zu reduzieren und die 
körperliche Fitness zu steigern. Bei möglicher begleitend medikamentös-toxischer Komponente durch eine systemische Wirkung der Elocom Crème, wurde die topische Kortikosteroidapplikation auf ein Minimum beschränkt. Bei der Verlaufskontrolle nach 6 Monaten zeigte sich eine erfolgreiche Gewichtsreduktion von $5 \mathrm{~kg}$ und eine Regredienz von Transaminasen und Ferritin.

Eine aktive Immunisierung gegen Hepatitis A und B war aufgrund der bereits vorhandenen serologischen Immunität bei unserem Patienten nicht erforderlich. 


\section{Fragen zur Nicht-alkoholischen Fettlebererkrankung}

\section{Frage 1}

Welche Aussage zur Prävalenz der NAFLD trifft zu?

a) Ungefähr $10 \%$ der Transaminasenerhöhungen sind auf eine NAFLD zurückzuführen.

b) Die NAFLD ist nach alkoholischen Fettlebererkrankungen und Virushepatitiden die dritthäufigste Hepatopathie.

c) Ungefähr 10-15\% der Bevölkerung sind von einer NAFLD betroffen.

d) Die Prävalenz ist bei Patienten mit DM Typ 2 deutlich höher als in der Allgemeinbevölkerung.

e) Die NAFLD ist ausschliesslich eine Erkrankung des höheren Lebensalters.

\section{Frage 2}

Welcher der folgenden Befunde ist passt nicht zu einer NAFLD?
a) Erhöhtes TSH
b) Leichte Erhöhung der antinukleären Antikörper
c) Normale Transaminasen
d) HBs-Antigen nachweisbar
e) Erhöhtes Serumferritin

Frage 3

Bei einem übergewichtigen, kardiopulmonal kompensierten Patienten mit einem D.M. Typ 2 fallen im Rahmen eines Routinelabors erhöhte Transaminasen auf. Welcher nächste diagnostische Schritt ist nicht sinnvoll?
a) Abdomensonographie
b) Anamnestische Erhebung des täglichen Alkoholkonsums
c) Echokardiographie zum Ausschluss einer Rechtsherzinsuffizienz mit Stauungshepatopathie
d) Erweiterte Labordiagnostik
e) Erhebung der Medikamentenanamnese

Frage 4

Was ist der diagnostische Goldstandard zur Diagnosesicherung und Prognoseabschätzung?
a) MRI
b) Fibroscan
c) Sonographie
d) Computertomographie
e) Leberbiopsie 
Welches der folgenden Medikamente kann bei NAFLD-Patienten ohne erhöhtes Hepatotoxizitätsrisiko eingesetzt werden?
a) Amiodaron
b) Prednison
c) Valproinsäure
d) Pravastatin
e) Methotrexat

\section{Autoren}

Dr. med. Christine Lang, Dr. med. Anna Georgi, Dr. med. Christoph Gubler

Klinik und Poliklinik für Innere Medizin, Klinik für Gastroenterologie, Universitätsspital Zürich

\section{Korrespondenzadresse}

Dr. med. Anna Georgi

Klinik und Poliklinik für Innere Medizin,

UniversitätsSpital Zürich

Rämistrasse 100

8091 Zürich

annakatharina.georgi@usz.ch

\section{Bibliographie}

Torres DM, Williams CD, Harrison SA. Features, Diagnosis, and Treatment of Nonalcoholic Fatty Liver Disease. Clin Gastroenterol Hepatol. 2012 Aug; 10(8):837-858.

Balmer ML, Dufour JF. Nicht-alkoholische Steatohepatitis - von NAFLD zu MAFLD. Therapeutische Umschau 2011; 68(4):183-188.

Bernsmeier C, Heim MH. Nicht-alkoholische Fettleber und Steatohepatitis. Schweiz Med Forum 2011; $11(4): 53-$ 57.

Ratziu V, Bellentani S, Cortez-Pinto H, Day C, Marchesini G. A position statement on NAFLD/NASH based on the EASL 2009 special conference. J Hepatol. 2010 Aug; 53(2):372-384.

Loria P, Adinolfi LE, Bellentani S, Bugianesi E, Grieco A, Fargion S, Gasbarrini A, Loguercio C, Lonardo A, Marchesini G, Marra F, Persico M, Prati D, Baroni GS. Practice guidelines for the diagnosis and management of nonalcoholic fatty liver disease. A decalogue from the Italian Association for the Study of the Liver (AISF) Expert Committee. Dig Liver Dis. 2010 Apr; 42(4):272-282.

Chalasani N, Younossi Z, Lavine JE, Diehl AM, Brunt EM, Cusi K, Charlton M, Sanyal AJ. The diagnosis and management of non-alcoholic fatty liver disease: practice Guideline by the American Association for the Study of Liver Diseases, American College of Gastroenterology, and the American Gastroenterological Association. Hepatology. 2012 Jun; 55(6):2005-2023. 


\section{Antworten zu den Fragen zur Nicht-alkoholischen Fettlebererkrankung aus PRAXIS Nr.}

\section{Frage 1}

\section{Richtig ist Antwort d)}

ad a) Ungefähr 25-30\% der Transaminasenerhöhungen sind auf die NAFLD zurückzuführen.

ad b) Die NAFLD ist die häufigste Hepatopathie mit weltweit steigender Prävalenz.

ad c) Die Prävalenz der Fettlebererkrankung liegt in der westlichen Gesamtbevölkerung bei etwa 20-30\%.

ad d) Bei bis zu 70\% der Patienten wird sonographisch eine Fettleber diagnostiziert

ad e) Patienten aller Altersklassen können an einer NAFLD leiden, die Prävalenz steigt mit zunehmendem Lebensalter.

\section{Frage 2}

\section{Richtig ist Antwort d)}

ad a) Ein erhöhtes TSH spricht für eine hypothyreote Stoffwechsellage. Eine Hypothyreose gehört zu den Komorbiditäten, welche mit einer NAFLD assoziiert sein können.

ad b) Milde Erhöhungen der antinukleären Antikörper (ANA) wurden bei einer signifikanten Patientenzahl mit einer NASH beobachtet und sind nicht spezifisch für eine autoimmune Hepatopathie.

ad c) In der Regel sind die Transaminasen bei NAFLD moderat erhöht (1-5 fach des oberen Normwertes, ALAT>ASAT). Obwohl die Höhe der Transaminasen häufig mit dem Ausmass der Entzündung oder Fibrose korreliert, können auch Patienten mit histologisch gesicherter Fettleber oder NASH normale Transaminasen haben. Normale Transaminasen schliessen eine NAFLD nicht aus.

ad d) Ein positives HBs-Antigen sichert die Diagnose einer chronischen Hepatitis und muss serologisch weiter abgeklärt werden. Eine begleitende NAFLD ist möglich, die Diagnose und Einschätzung der Relevanz kann aber in diesem Fall nur mittels Leberbiospie erfolgen.

ad e) Das Serumferritin ist bei Patienten mit einer NASH häufig erhöht. Eisen wird als Ferritin in der Leber gespeichert. Bei Entzündung und Destruktion von Hepatozyten wird dieses freigesetzt und das Ferritin im Serum steigt.

\section{Frage 3}

\section{Richtig ist Antwort c)}

ad a) Die Sonographie ist das Verfahren der Wahl zur Beurteilung der Lebermorphologie sowie zum Nachweis einer Steatose und sollte bei jedem Patienten mit einer unklaren Leberwerterhöhung durchgeführt werden.

ad b, e) Anamnestisch müssen Ursachen einer sekundären Leberverfettung, wie ein signifikanter Alkoholkonsum (> $20 \mathrm{~g} / \mathrm{d}$ bei Frauen, > $30 \mathrm{~g} / \mathrm{d}$ bei Männern) und hepatotoxische Medikamente, ausgeschlossen werden.

ad c) Eine Stauungshepatopathie mit Rechtsherzinsuffizienz kann zwar ebenfalls zu erhöhten Transaminasen führen, ist bei einem klinisch kompensierten Patienten aber eher unwahrscheinlich. Eine Echokardiographie sollte in einem zweiten Schritt erfolgen, sofern sich in der Abdomensonographie Zeichen einer Rechtsherzinsuffizienz finden (gestaute Lebervenen, fehlende Atemvariabilität der V. cava inf.).

ad d) Die erweiterte Labordiagnostik dient dem Ausschluss einer viralen, autoimmunen, hereditären oder metabolischen Ursache der Lebersteatose. 


\section{Frage 4}

\section{Richtig ist Antwort e)}

ad a, c, d) CT, MRI und Sonographie sind geeignete Untersuchungsmodalitäten zum Nachweis einer Lebersteatose. Das Verfahren der ersten Wahl ist dabei die Sonographie.

ad b) Der Fibroscan ist eine nicht-invasive Alternative zur Leberbiopsie zur Abschätzung des Fibrosegrades, welcher mit der gemessenen Lebersteifigkeit korreliert.

ad e) Goldstandard für die Diagnose und Prognoseabschätzung ist die Leberbiopsie, da sie als einziges Verfahren eine Differenzierung zwischen simpler Fettleber und NASH, sowie eine zuverlässige Erfassung des Fibrosegrades erlaubt.

\section{Frage 5}

\section{Richtig ist Antwort d)}

ad a-c, e) Amiodaron, Kortikosteroide, Valproinsäure und Methotrexat können zu einer medikamentöstoxischen Leberschädigung führen und Ursache einer sekundären Steatose sein.

ad d) Statine können auch bei Patienten mit einer NAFLD zur Therapie der Dyslipidämie ohne erhöhtes Hepatotoxizitätsrisiko eingesetzt werden. 